\title{
Characterisation of mildew resistant wheat-rye substitution lines and identification of an inverted chromosome by fluorescent in situ hybridisation
}

\author{
P-O Forsström ${ }^{1}$, A Merker ${ }^{1}$ and T Schwarzacher ${ }^{2}$ \\ ${ }^{1}$ Department of Crop Science, Swedish University of Agricultural Sciences, SE-230 53 Alnarp, Sweden; ${ }^{2}$ Department of Biology, \\ University of Leicester, Leicester, LE1 7RH, UK
}

\begin{abstract}
Seven different mildew resistant wheat lines derived from crosses between triticale and bread wheat were examined by molecular cytogenetics and chromosome C-banding in order to determine their chromosomal composition. Genomic in situ hybridisation (GISH) showed the presence of rye germplasm in all the lines and identified three substitution lines, three double substitution lines and one additionsubstitution line. C-banding identified rye chromosomes $1 \mathrm{R}$ and $4 \mathrm{R}$ in the addition-substitution line, rye chromosomes $1 R$ and $6 R$ in two substitution lines and $1 R$ and $2 R$ in the third line, and rye chromosome $1 \mathrm{R}$ in the three substitution
\end{abstract}

lines. Two of the latter lines (7-102 and 7-169) contained a modified form of the chromosome; fluorescent in situ hybridisation (FISH) using five different repetitive DNA-probes showed a pericentric inversion of $1 \mathrm{R}$ in both lines. The breakpoints of the $1 \mathrm{R}$ inversion were between (1) the $5 \mathrm{~S}$ rDNA site and the NOR-region on the satellite of the short arm, and (2) between two $\mathrm{AAC}_{(5)}$ sites close to the centromere on the long arm. The role of the rye chromosomes in the mildew resistance, the utilisation of the inverted $1 R$ and the significance of the lines in wheat breeding are discussed. Heredity (2002) 88, 349-355. DOI: 10.1038/sj/hdy/6800051

Keywords: molecular cytogenetics; C-banding; physical chromosome mapping; pericentric inversion; chromosomal substitution

\section{Introduction}

The rye (Secale cereale L.) genome has shown potential for improvement of bread wheat (Triticum aestivum L.), where wheat-rye substitutions and translocations have been and are frequently used in resistance breeding (Rabinovich, 1998) and the 1B/1R wheat-rye translocation is present in the highest yielding cultivars currently grown in Europe and Canada (Heslop-Harrison et al, 1990). A detailed analysis of the introgressed rye chromatin is essential for practical breeding. Molecular cytogenetics has proven to be an efficient method to determine the origin of genomes, chromosomes and parental genomes in hybrids (Schwarzacher et al, 1989). The advantage of genomic in situ hybridisation (GISH) is the recognition of all alien chromosome segments contained in the nucleus and is therefore the method of choice when interspecific crosses and derived introgressed lines are analysed to reveal alien chromosomes and translocations (Murata et al, 1992; Schwarzacher et al, 1992; Mukai et al, 1993; Taketa et al, 1997).

Fluorescent in situ hybridisation (FISH) enables physical mapping of repetitive DNA sequences to specific chromosomal sites. In both wheat and rye several such probes are cloned and can be used for identification of

Correspondence: P-O Forsström, Department of Crop Science, Swedish University of Agricultural Sciences, SE-230 53 Alnarp, Sweden. E-mail: Per-Olov.Forsstrom@vv.slu.se

Received 10 April 2001; accepted 9 November 2001 chromosomes in those genomes and their chromosomes (Mukai et al, 1993; Castilho and Heslop-Harrison, 1995; Cuadrado et al, 1995, 2000; Pedersen and Langridge, 1997; Cuadrado and Schwarzacher, 1998). Physical mapping of differentially labelled repetitive DNA sequences simultaneously in one experiment and in combination with total genomic DNA (eg Leitch et al, 1991; Mukai et al, 1993; Taketa, 1997) allows introgressed chromosomes or chromosome segments from alien species to be detected in wheat lines, identifying the chromosomes involved and determining the nature and organisation of any chromosome rearrangements.

This study was performed in order to identify and characterise rye chromosomes that were detected in mildew resistant wheat lines isolated in offsprings from triticale $\times$ wheat hybrids. GISH, DAPI staining and Cbanding was used to identify rye chromatin in the substitution and addition-substitution lines and FISH with repetitive probes to identify most wheat chromosomes and fine mapping the rearrangement detected in an aberrant chromosome found in two of the lines.

\section{Materials and methods}

\section{Plant material}

In populations derived from crosses between hexaploid triticale and bread wheat, lines with a broad mildew resistance were isolated (Forsström and Merker, 2001). Briefly, hexaploid winter triticales were crossed and 
backcrossed to mildew susceptible wheat cultivars, and then selfed. Screening for mildew resistance was performed by using three different mildew isolates. Seven lines were analysed in the present study: 1-17 in $\mathrm{F}_{7}$ and $7-73,7-102,7-169,7-225,7-317 \mathrm{a}$ and $7-317 \mathrm{~b}$ in $\mathrm{BC}_{1} \mathrm{~F}_{6}$ showed resistance to all isolates and all of them were fully fertile. These lines are maintained in Sweden at the Department of Crop Science in Alnarp at the Swedish University of Agricultural Sciences.

\section{C-banding}

Chromosome C-banding followed the method described by Gill et al (1991) and used excised root tips pre-treated with $0.05 \%$ colchicine for $3 \mathrm{~h}$ in room temperature followed by $24 \mathrm{~h}$ in ice water before fixation in 3:1 (v/v) ethanol:acetic acid.

\section{Preparation of chromosomes for in situ hybridisation}

Seed germination and chromosome preparation followed the technique as described by Schwarzacher et al (1989) and Schwarzacher and Heslop-Harrison (2000). Seedling roots were synchronised with 24 -h ice treatment before fixation, the cell wall material was digested with $3 \%$ $(\mathrm{w} / \mathrm{v})$ pectinase $($ Sigma, $450 \mathrm{u} / \mathrm{ml}), 1.8 \%(\mathrm{w} / \mathrm{v})$ cellulase (Calbiochem, $4000 \mathrm{u} / \mathrm{g})$ and $0.2 \% \quad(\mathrm{w} / \mathrm{v})$ cellulase (Onozuka RS, $5000 \mathrm{u} / \mathrm{g}$ ) and chromosome preparations made on glass slides by squashing in $45 \%$ and $60 \%$ acetic acid.

\section{Probes and labelling}

The following probes were used in this study: pTa71 contains a 9-kb EcoRI fragment of the repeat unit of 25S-5.8S$18 \mathrm{~S}$ rDNA isolated from $T$. aestivum (Gerlach and Bedbrock, 1979) and re-cloned in pUC19. The clone was linearised with HindIII before labelling. pTa794 contains a $410 \mathrm{bp} \mathrm{BamHI}$ fragment of the 5S rDNA isolated from $T$. aestivum (Gerlach and Dyer, 1980). pSc119.2 contains a $120 \mathrm{bp}$ tandem repeated DNA sequence isolated from $S$. cereale and subcloned by McIntyre et al (1990). pSc200 contains a $380 \mathrm{bp}$ tandem repeat sequence from $S$. cereale cloned in pUC18 (Vershinin et al, 1995). dpTa1 contains a tandem repeat with a monomeric length of $340 \mathrm{bp}$ isolated from T. aestioum and subcloned by Vershinin et al (1994). This probe is D-genomic and homologous to pAs1 (Rayburn and Gill, 1986). $\mathrm{AAC}_{(5)}$ is a synthetic oligonucleotide sequence (Genosys). Total genomic DNA rye 'Petkus' was sheared to $5-8 \mathrm{~kb}$ pieces by autoclaving. For labelling, biotin-16-dUTP and digoxigenin-16-dUTP (Roche Diagnostics) were incorporated in separate reactions. pTa71 and total genomic rye DNA were labelled by nick translation. pSc119.2, pSc200, pTa794 and dpTa1 were labelled by PCR using the universal forward and reverse $\mathrm{M} 13$ sequencing primers. $\mathrm{AAC}_{(5)}$ was labelled by random priming (Cuadrado et al, 2000).

\section{In situ hybridisation}

Probe mixture, DNA:DNA in situ hybridisation and detection followed the method described by Schwarzacher and Heslop-Harrison (2000). The probe mixture contained $50 \%(\mathrm{v} / \mathrm{v})$ formamide, $20 \%(\mathrm{w} / \mathrm{v})$ dextran sulphate, $2 \times$ SSC, 25-100 ng probe, $20 \mu \mathrm{g}$ of salmon sperm DNA and $0.3 \%$ sodium dodecyl sulfate (SDS), and in some experiments autoclaved total genomic DNA from wheat cv. 'Chinese Spring' as blocking DNA at 4$20 \times$ probe concentration. Hybridisation took place over night at $37^{\circ} \mathrm{C}$ and the most stringent wash was carried out with $20 \%(\mathrm{v} / \mathrm{v})$ formamide and $0.1 \times \mathrm{SSC}$ at $42^{\circ} \mathrm{C}$. Hybridisation sites were detected with $2.0 \mu \mathrm{g} / \mathrm{ml}$ streptavidin conjugated to Alexa594 (Molecular Probes) or $3.0 \mu \mathrm{g} / \mathrm{ml}$ streptavidin conjugated to Cy3 (Sigma) and $4 \mu \mathrm{g} / \mathrm{ml}$ antidigoxigenin conjugated to flourescein isothiocyanate (FITC) (Roche Diagnostics). Chromosomes were counterstained with $0.2 \mu \mathrm{g} / \mathrm{ml}$ DAPI (4',6-diamidino-2phenylindole) diluted in McIlvaines buffer $\mathrm{pH} 7$ and mounted in antifade solution (Citiflour).

\section{Photography and analysis}

Photographs of C-banding were taken with Kodak technical pan black and white film, and in situ hybridisation with Fujicolor SuperHG400 colour film. Negatives were scanned to CD-rom by Kodak Digital Science. Colour figures were prepared using Adobe Photoshop (Adobe Systems) with only those processing functions that are equally applied to all pixels of the image.

For each line, 15 metaphases were analysed after Giemsa C-banding following the karyotypes published by Sybenga (1983) and Gill et al (1991), 10 metaphases after DAPI staining and in at least four complete and partial metaphases for each of the genomic rye and repetitive in situ hybridisation probes. Detailed signal of the derivative $1 R$ (der1R) was analysed in a total of 50 individual chromosomes hybridised with various probe combinations and the combined signals superimposed on the published chromosome morphology of chromosome 1R (Cuadrado et al, 1995) in Figure 3.

\section{Results}

C-banding of the progeny of a cross between a mildew resistant triticale line and susceptible bread wheat, identified one addition-substitution line containing rye chromosomes $1 \mathrm{R}$ and $4 \mathrm{R}$, three double wheat-rye substitution lines containing rye chromosomes $1 \mathrm{R}$ and $6 \mathrm{R}$ (Figure 1a) or chromosomes $1 \mathrm{R}$ and $2 \mathrm{R}$, a wheat-rye substitution line with the presence of rye chromosome $1 \mathrm{R}$ and two wheatrye substitution lines with der1R (Figure 1b). GISH (Table 1 and Figure $2 b, f$ ) showed the presence of rye chromatin in the six investigated lines and FISH with repetitive probes was used in five of the lines (Table 1 and Figure $2 d, g-n$ ), which gave specific information about the identity of chromosomes and chromosome segments. The results are summarised in Table 1 and are briefly described below.

\section{Line 1-17}

Line 1-17 was an addition-substitution line $(2 n=44)$ with the presence of the rye chromosomes $1 \mathrm{R}$ and $4 \mathrm{R}$ as detected by C-banding. GISH confirmed the presence of four rye chromosomes and DAPI-staining the presence of $1 \mathrm{R}$. This line had small seeds and was relatively weak.

\section{Line 7-73}

Line $7-73$ was a double substitution line $(2 n=42)$ and the rye chromosomes $1 \mathrm{R}$ and $6 \mathrm{R}$ were identified both with $\mathrm{C}$-banding and DAPI (Figure 2a). The presence of four rye chromosomes was also detected by GISH (Figure $2 b$ ). The NORs were identified by the probe pTa71 and revealed the presence of $1 \mathrm{R}, 1 \mathrm{~B}, 6 \mathrm{~B}, 1 \mathrm{~A}$ and 5D (Table 1). The hybridisation pattern with probe pSc119.2 confirmed the presence of $6 \mathrm{R}$. The probe $\mathrm{dpTa} 1$ revealed that the 


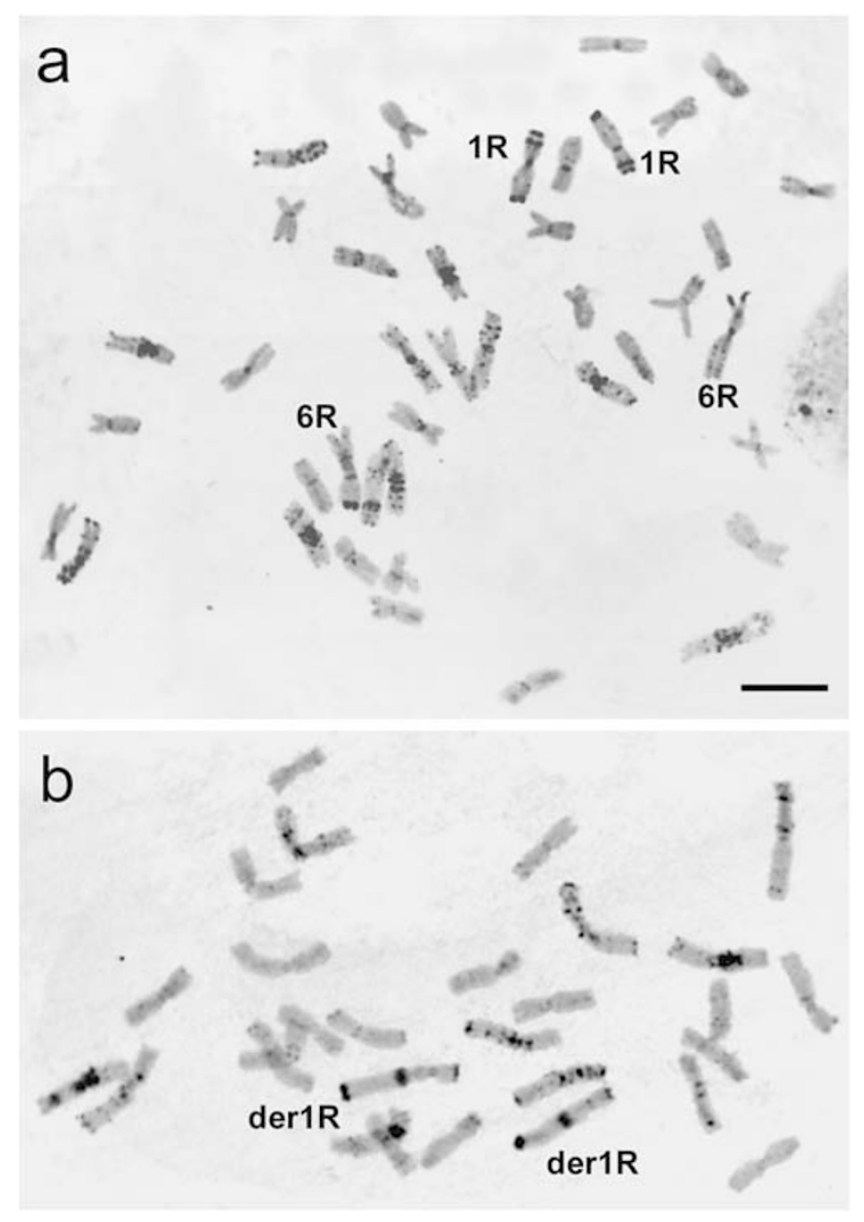

Figure $1 \mathrm{C}$-banded root tip methaphase chromosomes. (a) A double ( $1 R$ and $6 R$ ) wheat-rye substitution line $(2 n=42)$. (b) A partial methaphase of line 7-102 showing the der1R chromosomes with unequal arm ratio and a strong C-band on the long arm. Bar equals $10 \mu \mathrm{m}$. line lacked four D-chromosomes. It is most likely that the line had the chromosomal composition 1R(1D) and 6R(6D).

\section{Line 7-317a}

Line 7-317a was a substitution line $(2 n=42)$ and C-banding revealed the presence of rye chromosomes $1 \mathrm{R}$ and $2 \mathrm{R}$ (Table 1). This line was not analysed by in situ hybridisation.

\section{Line $7-317 b$}

Line $7-317 \mathrm{~b}$ was a substitution line $(2 n=42)$ and C-banding identified the rye chromosomes $1 \mathrm{R}$ and $6 \mathrm{R}$, while GISH showed the presence of four rye chromosomes. Hence this line (7-317) consisted of two different sub lines, and the $6 \mathrm{R}$ sub line, 7-317b, was studied with FISH. The probe pTa71 detected the presence of $1 \mathrm{R}, 1 \mathrm{~B}, 6 \mathrm{~B}, 1 \mathrm{~A}$ and $5 \mathrm{D}$. The probe dpTa1 identified the presence of 12 D-genome chromosomes. The rye chromosome $1 R$ had substituted 1D while 6R had substituted 6A.

\section{Line 7-225}

Line $7-225$ was a substitution line $(2 n=42)$ and rye chromosome $1 \mathrm{R}$ was identified by C-banding and DAPI-staining (Figure 2c). GISH confirmed the presence of two rye chromosomes. Probe pTa71 identified six major NORs and four minor NORs, which indicated the presence of $1 \mathrm{R}, 1 \mathrm{~B}, 6 \mathrm{~B}$ and $1 \mathrm{~A}, 5 \mathrm{D}$ (Table 1). The D-genome specific probe dpTa1 showed the presence of 12 D-chromosomes (Figure $2 \mathrm{~d}$ ). We concluded that $1 \mathrm{D}$ was replaced by $1 \mathrm{R}$ in a $1 R(1 D)$ substitution.

\section{Line 7-102}

This line was a substitution line $(2 n=42)$ as determined by C-banding (Figure 1b) and DAPI-staining (Figure 2e). C-banding showed an unusual chromosome pair with subtelomeric bands and a strong heterochromatic band $1 / 3$ of the way down the long arm (Figure $1 \mathrm{~b}$ ). This latter band was similar in size with the NOR of the short arm of the normal 1R (compare Figure 1b with Figure 1a). GISH

Table 1 Chromosome number and identification of the introgressed rye chromosomes using C-banding and fluorescent in situ hybridisation with total genomic rye DNA, cloned and synthetic probes

\begin{tabular}{|c|c|c|c|c|c|c|c|c|c|}
\hline Line & C-band & Genomic rye & $p T a 71$ & pTa794 & pSc119.2 & $p S c 200$ & $A A C$ & $d p T a 1$ & $D A P 1$ \\
\hline $1-17$ & $\begin{array}{l}2 n=44 \\
1 \mathrm{R}, 4 \mathrm{R}\end{array}$ & $4 \mathrm{chrs}$ & - & - & - & - & - & - & $1 \mathrm{R}$ \\
\hline $7-73$ & $\begin{array}{l}2 n=42 \\
1 R, 6 R\end{array}$ & $4 \mathrm{chrs}$ & $\begin{array}{c}1 \mathrm{R}, 1 \mathrm{~B}, 6 \mathrm{~B}, 1 \mathrm{~A} \\
5 \mathrm{D}\end{array}$ & - & 1R? 6R & - & - & $10 \mathrm{D}$-chrs & $1 \mathrm{R}, 6 \mathrm{R}$ \\
\hline $7-317 a$ & $\begin{array}{l}2 n=42 \\
1 R, 2 R\end{array}$ & - & - & - & - & - & - & - & - \\
\hline $7-317 \mathrm{~b}$ & $\begin{array}{l}2 n=42 \\
1 R, 6 R\end{array}$ & $4 \mathrm{chrs}$ & $\begin{array}{c}1 \mathrm{R}, 1 \mathrm{~B}, 6 \mathrm{~B}, 1 \mathrm{~A} ? \\
5 \mathrm{D} ?\end{array}$ & - & $6 \mathrm{R}$ & - & - & 12 D-chrs & $1 R, 6 R$ \\
\hline $7-225$ & $\begin{array}{c}2 n=42 \\
1 \mathrm{R}\end{array}$ & $2 \mathrm{chrs}$ & $\begin{array}{c}1 \mathrm{R}, 1 \mathrm{~B}, 6 \mathrm{~B}, 1 \mathrm{~A} ? \\
5 \mathrm{D} ?\end{array}$ & - & - & - & - & 12 D-chrs & $1 \mathrm{R}$ \\
\hline $7-102$ & $\begin{array}{c}2 n=42 \\
1 \mathrm{R} ?\end{array}$ & $2 \mathrm{chrs}$ & $\begin{array}{c}\text { der } 1 \mathrm{R}, 1 \mathrm{~B}, 6 \mathrm{~B} \\
1 \mathrm{~A}, 5 \mathrm{D}\end{array}$ & der $1 R$ & der $1 R$ & der $1 R$ & $\begin{array}{c}\text { der } 1 \mathrm{R} \\
14 \mathrm{~B}-\mathrm{chrs}\end{array}$ & 12 D-chrs & der $1 R$ \\
\hline $7-169$ & $\begin{array}{c}2 n=42 \\
1 \mathrm{R} ?\end{array}$ & $2 \mathrm{chrs}$ & $\begin{array}{c}\text { der } 1 R, 1 B, 6 B \\
1 A, 5 D\end{array}$ & der $1 R$ & - & der $1 R$ & - & 12 D-chrs & der $1 R$ \\
\hline
\end{tabular}



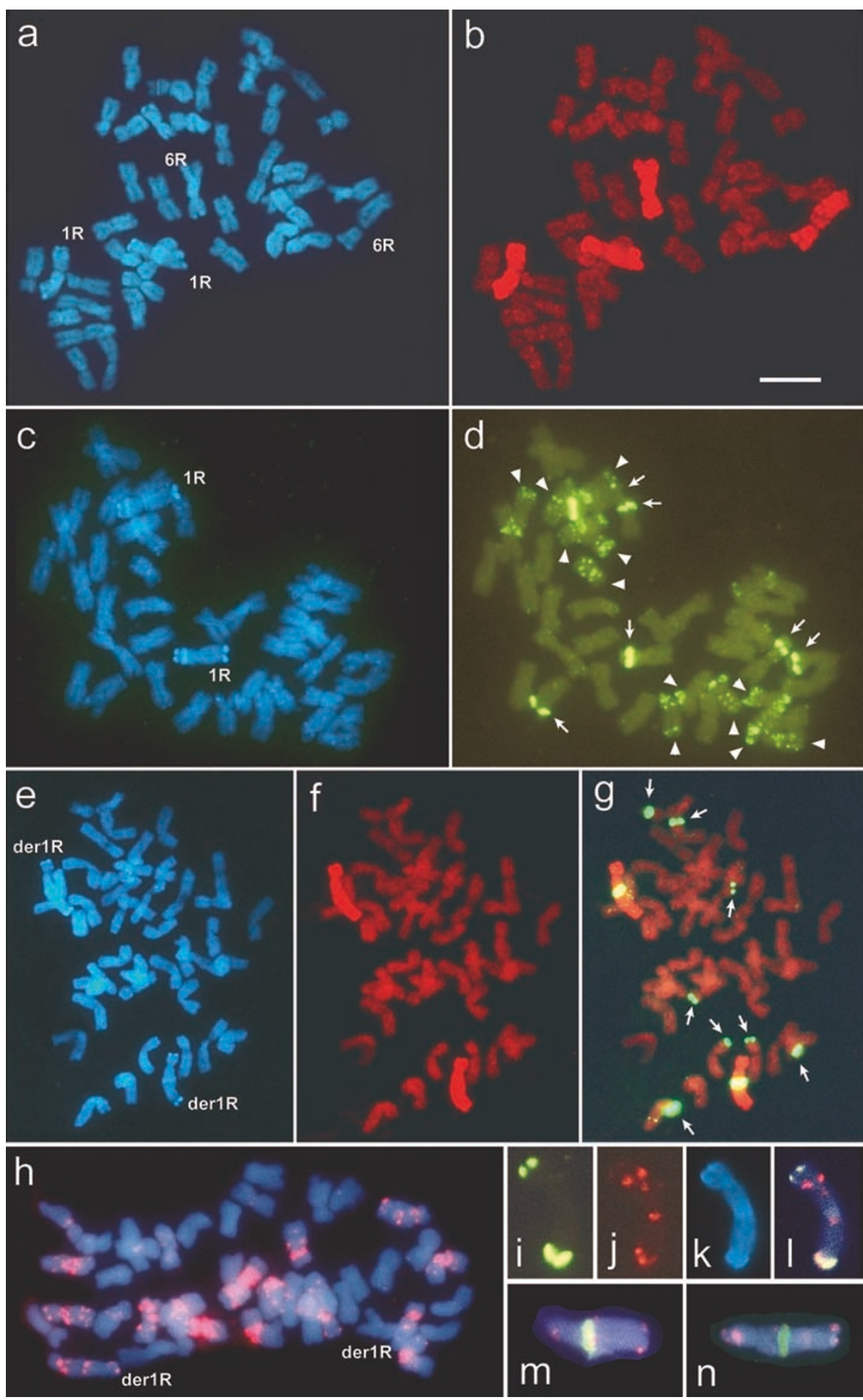

Figure 2 Photomicrographs of DAPI staining and fluorescent in situ hybridisation with total genomic rye DNA and cloned or synthetic probes to metaphases of mildew resistant wheat lines incorporating rye chromatin. (a, b) Line 7-73 contains rye chromosome pairs $1 \mathrm{R}$ and 6R that show subtelomeric bands with DAPI (a) and are labelled strongly with total genomic DNA from rye (b). (c, d) Line 7-225: after DAPI staining chromosome 1R can be identified by its three heterochromatic bands. Probes pTa71 and dpTa1, both labelled with digoxigenin and detected with yellow-green fluorescence, identified the larger NOR bands on chromosomes 1R, 1B and 6B (arrows); and 12 D-genome chromosomes with several small interstitial bands (arrow heads). (e-g) Line 7-102: the same metaphase probed with rye genomic DNA (red in $\mathbf{f}$ and $\mathbf{g}$ ) and pTa71 (yellow-green in $\mathbf{g}$ ) and stained with DAPI (blue in e). The der chromosome 1R has a very unequal arm ratio and is characterised by a large pTa71 site in the long arm. Eight further sites of pTa71 are detected on the wheat chromosomes (arrows in g). (h) Line 7-102: the probe (AAC) $)_{5}$ hybridises to all B genome chromosomes, chromosome $4 \mathrm{~A}$ and identifies four significant bands on

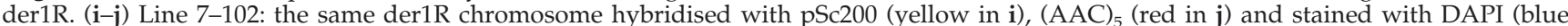
in $\mathbf{k}$ ). The overlay in (1) shows the relation of the probes and DAPI bands. (m) Line 7-102: chromosome der1R probed with pTa71 (yellow) and $(\mathrm{AAC})_{5}$ (red) and stained with DAPI (blue). (n) Line 7-102: chromosome der1R probed with pTa71 (yellow) and pSc119.2 (red) and stained with DAPI (blue). Bar equals $12 \mu \mathrm{m}$ in $\mathbf{a}-\mathbf{d}$ and $\mathbf{h} ; 10 \mu \mathrm{m}$ in $\mathbf{e}-\mathbf{g}$ and $17.5 \mu \mathrm{m}$ in $\mathbf{i}-\mathbf{n}$. 
showed that entirely rye chromatin was contained in the unusual chromosome (Figure 2f) and no other translocation either with wheat or rye chromosomes seemed to have contributed to the rearrangement.

In situ hybridisation with pTa71 confirmed the presence of the NOR in the intercalary position of the long arm, identifying the aberrant chromosome as a der1R. pTa71 also showed the four major and the four minor NORs of the wheat chromosomes 1B, 6B, 1A and 5D. The probe dpTa1 determined the presence of 12 D-genome chromosomes (Table 1) supporting the presence of a 1R(1D) substitution. Probe pSc200 hybridises to both subtelomeric regions of $1 \mathrm{R}$ (Vershinin et al, 1995) and it hybridised at the same positions in der1R (Figure 2i). Similarly, the location of the 5S rDNA as detected with the probe pTa794 was unaltered at the distal end of the short arm (Figure 3). The probe pSc119.2 hybridised at both subterminal regions and at an intercalary position in both arms of the normal 1R (Cuadrado et al., 1995). Both subterminal bands and the intercalary band of the long arm were detected at normal sites in der1R, while a second intercalary band was found in the long arm, proximal to the NOR (Figure $2 \mathrm{n}$ and 3 ). The probe $\mathrm{AAC}_{(5)}$ detected paracentromeric sites on both chromosome arms of the normal $1 \mathrm{R}$, the one of the long arm being slightly stronger than the one on the short arm, in addition to a proximal intercalary and a subtelomeric site on the long arm (Cuadrado and Schwarzacher, 1998). The subtelomeric site was located proximally to the pSc119.2 site and was found in the same position in the der1R chromosome (Figures $2 \mathrm{~h}-\mathrm{m}$ and 3 ). The paracentromeric sites were rearranged, the one on the short arm now being stronger (eg Figure $2 \mathrm{j}$ ) and the interstitial site was distally located relative to the NOR-region.

\section{Line 7-169}

This line was a substitution line $(2 n=42)$ as determined by C-banding (Table 1) containing the aberrant chromosome der1R. Line 7-169 seemed to be identical to line 7-
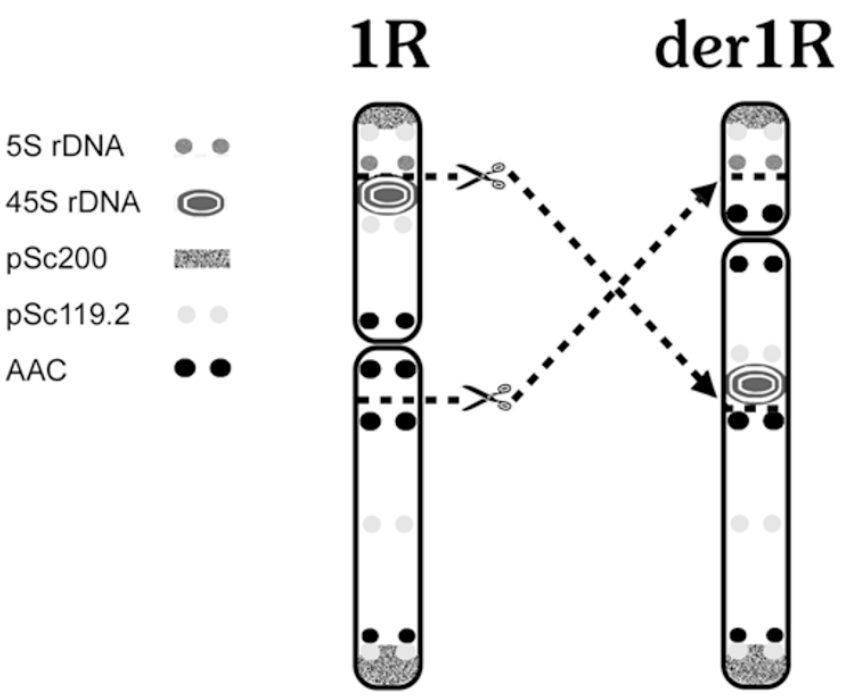

Figure 3 Schematic diagram of normal chromosome 1R and chromosome der1R and proposed breakpoints of the pericentric inversions. Chromosome morphology and band distribution was taken from Cuadrado et al (1995), Castilho and Heslop-Harrison (1995) and Cuadrado and Schwarzacher (1998).
102 (compare lines 7-102 and 7-169 in Table 1), as far as the rye chromosomes was concerned, which could be derived from a single meiotic event.

\section{Chromosome der1R}

From interpretation of all the FISH results we concluded that the der1R is a pericentric inversion with the breakpoints (1) between the 5S rDNA and the 45S rDNA site on the short arm and (2) between the paracentromeric and proximal interstitial site of $\mathrm{AAC}_{(5)}$ on the long arm, transferring the NOR-region and a pSc119.2 site to the long arm that now is $75 \%$ of the chromosome (Figure 3 ). From comparing the overall lengths of the chromosome, the size of hybridisation signals and distances between the different sites it is most likely that no $1 \mathrm{R}$ chromatin material was lost during the rearrangement and that no material from wheat or other rye chromosomes was incorporated.

\section{Discussion}

The present study using C-banding (Figure 1) and FISH (Figure 2) has shown the disomic presence of rye chromosomes in all the investigated wheat lines. Rye chromosome $1 \mathrm{R}$ was found in all the lines, as the only rye chromosome in three lines and together with either of the rye chromosomes $2 R, 4 R$ or $6 R$ in the other lines (Table 1). Use of repetitive probes to identify most of the wheat chromosomes allowed us to determine that chromosome $1 \mathrm{R}$ replaced chromosome $1 \mathrm{D}$ in all investigated lines, that chromosome $6 \mathrm{R}$ replaces $6 \mathrm{~A}$ in one and $6 \mathrm{D}$ in another line, and that chromosome $4 \mathrm{R}$ is present as an addition.

Rye chromosomes 1, 2, 4 and 6 are known carriers of mildew resistance (Driscoll and Jensen, 1965; Zeller, 1973; Lind, 1982; Heun et al, 1990; Friebe et al, 1994) and our resistant lines are the result of a selection for a broad mildew resistance to three different isolates during the development of a pre-breeding material from different sources of the entire rye genome. While chromosome $1 \mathrm{R}$ is regarded as the major contributor (Zeller, 1973), the involvement in the defence reaction and nature of resistance from the other rye chromosomes remains to be investigated.

Aberrant forms of chromosomes have not been reported extensively in wheat alien lines, although they are probably more commonly occurring than indicated by documented cases (Taketa et al, 1997). C-banding could identify the presence of a structural aberrant chromosome, while it was necessary to use detailed and comprehensive molecular cytogenetic methods to describe the aberrant chromosome as a pericentric inversion of rye chromosome 1R (Figure 3). GISH suggested that the chromosome is composed of rye chromatin (Figure $2 \mathrm{~g}$ ) while the use of five different repetitive DNA sequences with distinct distribution patterns on rye chromosomes (Cuadrado et al, 1995; Cuadrado and Schwarzacher, 1998) detected the inversion and localised the inversion breakpoints on the long and short arm (Figure 3). As the original $1 \mathrm{R}$ and the der1R shared the same number of hybridisation sites and distances between the sites, albeit their order being reversed in the middle of the chromosome, it is unlikely that material from other rye or wheat chromosomes is involved in the rearrangement.

The der1R in the lines 7-102 and 7-169 showed the 
same pattern of resistance as found in line 7-225, which had a normal 1R(1D) and derived from the same population, without any obvious effects on the resistance caused by the inversion. GISH excluded the presence of rye segments other than $1 \mathrm{R}$ in all the three substitution lines making $1 \mathrm{R}$, normal or derivative, the sole source of the acquired mildew resistance. The lines with the der1R are more difficult to maintain in crosses with other lines carrying $1 \mathrm{R}$ and contain no value of increased resistance over the original wheat cultivar per se. Instead, they could be used for the development of recombinant wheat-rye lines. These recombinant lines are particularly desired by plant breeders to retain useful genes while minimising the number of deleterious characters from the alien species by reducing the size of the incorporated alien chromatin (Koebner et al, 1986). Several strategies have been developed to induce recombination such as using ionising radiation, through manipulation of the genetic control of homoeologous chromosome pairing or generating secondary recombinants by crosses between two lines sharing homologous regions of different length (see Koebner et al, 1986; Islam and Shepherd, 1991; Rogowsky et al, 1991). In combinations with normal or rearranged $1 \mathrm{R}$, the two lines described here could be used to create duplications or deletions of certain defined chromosome regions. Merker and Forsström (2000) described another structural rearrangement of chromosome $1 \mathrm{R}$ and crosses between such lines could create a diversity of progeny with duplicated and deleted chromosome segments and specific genes of $1 \mathrm{R}$ transferred to the wheat genome.

Physical and genetical mapping, besides understanding the organisation and evolution of genomes (HeslopHarrison, 2000), have substantially increased the potential to enhance the efficiency of plant breeding. Correlation of physical and genetic maps of markers and genes can be achieved by in situ hybridisation and identifies regions of the genome rich in genes and recombination to be targeted in breeding programmes (Schwarzacher, 1996). In the present study, we showed that FISH was efficient in describing aberrant chromosomes. Selection of plant material could therefore be improved by means of molecular cytogenetic methods and different traits more accurately traced through the breeding programmes for the benefit of targeting the goals more exactly in plant breeding.

\section{Acknowledgement}

This research was supported by a travel grant from the Royal Swedish Academy of Agriculture and Forestry, and carried out at the John Innes Centre, Norwich.

\section{References}

Castilho A, Heslop-Harrison JS (1995). Physical mapping of $5 S$ and 18S-25S rDNA and repetitive DNA sequences in Aegilops umbellulata. Genome 38: 91-96.

Cuadrado A, Ceoloni C, Jouve N (1995). Variation in highly repetitive DNA composition of heterochromatin in rye studied by fluorescence in situ hybridization. Genome 38: 10611069.

Cuadrado A, Schwarzacher T (1998). The chromosomal organization of simple sequence repeats in wheat and rye genomes. Chromosoma 107: 587-594.

Cuadrado A, Schwarzacher T, Jouve N (2000). Identification of different chromatin classes in wheat using in situ hybridiz- ation with simple sequence repeat oligonucleotides. Theor Appl Genet 101: 711-717.

Driscoll CJ, Jensen NF (1965). Release of a wheat-rye translocation stock involving leaf rust and powdery mildew resistances. Crop Sci 5: 279-280.

Friebe B, Heun M, Tuleen N, Zeller FJ, Gill BS (1994). Cytogenetically monitored transfer of powdery mildew resistance from rye into wheat. Crop Sci, 34: 621-625.

Forsström PO, Merker A (2001). Sources of wheat powdery mildew resistance from wheat-rye and wheat-Leymus hybrids. Hereditas 134: 115-119.

Gerlach WL, Bedbrock JR (1979). Cloning and characterization of ribosomal RNA genes from wheat and barley. Nucl Acids Res 7: 1869-1885.

Gerlach WL, Dyer TA (1980). Sequence organization of the repeating units in the nucleus of wheat which contain 5S rRNA genes. Nucl Acids Res 8: 4851-4865.

Gill BS, Friebe B, Endo TR (1991). Standard karyotype and nomenclature system for description of chromosome bands and structural aberrations in wheat (Triticum aestivum). Genome 34: 830-839.

Heslop-Harrison JS (2000). Comparative genome organization in plants: from sequence and markers to chromatin and chromosomes. Plant Cell 12: 617-635.

Heslop-Harrison JS, Leitch AR, Schwarzacher T, AnamthawatJónsson K (1990). Detection and characterization of 1B/1R translocations in hexaploid wheat. Heredity 65: 385-392.

Heun M, Friebe B, Bushuk W (1990). Chromosomal location of the powdery mildew resistance gene of Amigo wheat. Phytopathology 80: 1129-1133.

Islam AKMR, Shepherd KW (1991). Production of wheat-barley recombinant chromosomes through induced homoeologous pairing. 1. Isolation of recombinants involving barley arm 3HL and 6HL. Theor Appl Genet 83: 489-494.

Koebner RMD, Shepherd KW, Appels R (1986). Controlled introgression to wheat of genes from rye chromosome arm 1 RS by induction of allosyndesis. 2. Characterisation of recombinants. Theor Appl Genet 73: 209-217.

Leitch IJ, Leitch AR, Heslop-Harrison JS (1991). Physical mapping of plant DNA sequences by simultaneous in situ hybridization of two differently labelled fluorescent probes. Genome 34: 329-333.

Lind V (1982). Analysis of the resistance of wheat-rye addition lines to powdery mildew of wheat (Erysiphe graminis f. sp. tritici). Tagungsber Akad Landwirtsch Wiss DDR 198: 509-520.

McIntyre CL, Pereira S, Moran LB, Appels R (1990). New Secale cereale (rye) DNA derivatives for the detection of rye chromosome segments in wheat. Genome 33: 635-640.

Merker A, Forsström PO (2000). Isolation of mildew resistant wheat-rye translocation lines from a double substitution line. Euphytica 115: 167-172.

Mukai Y, Nakahara Y, Yamamota M (1993). Simultaneous discrimination of the three genomes in hexaploid wheat by multicolor fluorescence in situ hybridization using total genomic and highly repeated DNA probes. Genome 36: 489-494.

Murata M, Nakata N, Yasumuro Y (1992). Origin and molecular structure of a midget chromosome in a common wheat carrying rye cytoplasm. Chromosoma 102: 27-31.

Pedersen C, Langridge P (1997). Identification of the entire chromosome complement of breadwheat by two-colour FISH. Genome 40: 589-593.

Rabinovich SV (1998). Importance of wheat-rye translocations for breeding modern cultivars of Triticum aestivum L. Euphytica 100: 323-340.

Rayburn AL, Gill BS (1986). Isolation of a D-genome specific repeated DNA sequence from Aegilops squarrosa. Plant Mol Biol Rep 4: 102-109.

Rogowsky PM, Guidet FLY, Langridge P, Shepherd KW, Koebner RMD (1991). Isolation and characterization of wheatrye recombinants involving chromosome arm 1DS of wheat. Theor Appl Genet 82: 537-544. 
Schwarzacher T (1996). The physical organization of Triticeae chromosomes. In: Heslop-Harrison JS (ed) Unifying Plant Genomes, Symposia of the Society for Experimental Biology, Number 50, The Company of Biologists Ltd: Cambridge, pp 71-75.

Schwarzacher T, Leitch AR, Bennett MD, Heslop-Harrison JS (1989). In situ localization of parental genomes in a wide hybrid. Ann Bot 64: 315-324.

Schwarzacher T, Anamthawat-Jónsson K, Harrison GE, Islam AKMR, Jia JZ, King IP, et al (1992). Genomic in situ hybridization to identify alien chromosomes and chromosome segments in wheat. Theor Appl Genet 84: 778-786.

Schwarzacher T, Heslop-Harrison JS (2000). Practical in situ hybridization. BIOS Scientific Publishers Ltd.

Sybenga J (1983). Rye chromosome nomenclature and homoeology relationships, workshop report. Z Pflanzenzucht 90: 297304.
Taketa S, Nakazaki T, Schwarzacher T, Heslop-Harrison JS (1997). Detection of a 4DL chromosome segment translocated to rye chromosome 5R in an advanced hexaploid triticale line Bronco 90. Euphytica 97: 91-96.

Vershinin A, Svitashev S, Gummeson P-O, Salomon B, Bothmer R Von, Bryngelsson T (1994). Characterization of a family of tandemly repeated DNA sequences in Triticeae. Theor Appl Genet 89: 217-225.

Vershinin AV, Schwarzacher T, Heslop-Harrison JS (1995). The large-scale genomic organization of repetitive DNA families at the telomeres of rye chromosomes. The Plant Cell 7: 18231833.

Zeller FJ (1973). 1B/1R wheat-rye chromosome substitutions and translocations. In: Proceedings of the 4th International Wheat Genetics Symposium, pp 209-222. 